\title{
On Einstein: Kaehlerian Recurrent and Symmetric Spaces of Second Order
}

\author{
K.S. Rawat, Nitin Uniyal and Mukesh Kumar \\ Department Of Mathematics H.N.B. Garhwal University Campus Badshahi Thaul , Tehri (Garhwal) - \\ 249199uttarakhand, India
}

\begin{abstract}
Roter (1964) and Matsumoto (1969) have studied on second order recurrent spaces and Kaehlerian spaces with parallel or vanishing Bochner curvature tensor. Singh (1972) have defined and studied Kaehlerian recurrent and Ricci- recurrent spaces of second order.

Further, Rawat and Prasad (2010) studied on holomorphically projectively flat parabolically Kaehlerian spaces.

In the present paper, we have studied Einstein - Kaehlerian recurrent and symmetric spaces of second order and several theorems have been derived within. The necessary and sufficient condition for an Einstein Kaehlerian conharmonic recurrent space to be Kaehlerian recurrent space is discussed.
\end{abstract}

Key words: Recurrent, symmetric, Kaehlerian spaces.

\section{Introduction:}

An $n=(2 m)$ dimensional Kaehlerian space is a Riemannian space which admits a tensor field $F_{i}^{h}$ satisfying the following condition (Yano 1965)

$$
\begin{array}{cc} 
& F_{j}^{h} F_{h}^{i}=-\delta_{j}^{i} \\
& F_{i j}=-F_{j i},\left(F_{i j}=F_{i}^{a} g_{a j}\right) \\
\text { and } \quad & F_{i, j}{ }^{h}=0,
\end{array}
$$

where the comma (,) followed by an index denotes the operator of covariant differentiation with respect to the metric tensor $g_{i j}$ of the Riemannian space .

The Riemannian curvature tensor $R_{i j k}^{h}$ is given by

$$
R_{i j k}^{h}=\partial_{i}\left\{\begin{array}{l}
h \\
j k
\end{array}\right\}-\partial_{j}\left\{\begin{array}{c}
h \\
i k
\end{array}\right\}+\left\{\begin{array}{c}
h \\
i a
\end{array}\right\}\left\{\begin{array}{c}
a \\
j k
\end{array}\right\}-\left\{\begin{array}{c}
h \\
j a
\end{array}\right\}\left\{\begin{array}{c}
a \\
i k
\end{array}\right\},
$$

where $\partial_{i}=\frac{\partial}{\partial x^{i}}$ and $\left\{x^{i}\right\}$ denotes real local co-ordinates.

The Ricci- tensor and the scalar curvature are given by

$$
R_{i j}=R_{a i j}^{a} \quad \text { and } \quad R=R_{i j} g^{i j} \quad \text { respectively. }
$$

The holomorphically conformal (Bochner) curvature tensor and Kaehlerian conharmonic curvature tensor are respectively given by

$$
\begin{aligned}
& K_{i j k}^{h}=R_{i j k}^{h}+\frac{1}{(n+4)}\left(R_{i k} \delta_{j}^{h}-R_{j k} \delta_{i}^{h}+\right. g_{i k} R_{j}^{h}-g_{j k} R_{i}^{h}+S_{i k} F_{j}^{h} \\
&\left.\quad-S_{j k} F_{i}^{h}+F_{i k} S_{j}^{h}-F_{j k} S_{i}^{h}+2 S_{i j} F_{k}^{h}+2 F_{i j} S_{k}^{h}\right) \\
&-\frac{R}{(n+2)(n+4)}\left(g_{i k} \delta_{j}^{h}-g_{j k} \delta_{i}^{h}+F_{i k} F_{j}^{h}-F_{j k} F_{i}^{h}+2 F_{i j} F_{k}^{h}\right)
\end{aligned}
$$

and

$$
\begin{aligned}
T_{i j k}^{h}=R_{i j k}^{h}+\frac{1}{(n+4)}\left(R_{i k} \delta_{j}^{h}-R_{j k} \delta_{i}^{h}+\right. & g_{i k} R_{j}^{h}-g_{j k} R_{i}^{h}+S_{i k} F_{j}^{h} \\
& \left.-S_{j k} F_{i}^{h}+F_{i k} S_{j}^{k}-F_{j k} S_{i}^{h}+2 S_{i j} F_{k}^{h}+2 F_{i j} S_{k}^{h}\right),
\end{aligned}
$$

where $S_{i j}=F_{i}^{a} R_{a j}$

Let us suppose that a Kaehlerian space is an Einstein- one, then the Ricci tensor satisfies

$$
R_{i j}=\frac{R}{n} g_{i j} ; R_{, a}=0 .
$$

from which we obtain

$$
R_{i j, a}=0, S_{i j, a}=0 \text { and } S_{i j}=\frac{R}{n} F_{i j}
$$

If a Kaehlerian space is an Einstein one, then the Bochner curvature tensor and the Kaehlerian Conharmonic curvature tensor respectively reduces to the forms:

$$
V_{i j k}^{h}=R_{i j k}^{h}+\frac{R}{n(n+2)}\left(g_{i k} \delta_{j}^{h}-g_{j k} \delta_{i}^{h}+F_{i k} F_{j}^{h}-F_{j k} F_{i}^{h}+2 F_{i j} F_{k}^{h}\right)
$$


and $\quad A_{i j k}^{h}=R_{i j k}^{h}+\frac{2 R}{n(n+4)}\left(g_{i k} \delta_{j}^{h}-g_{j k} \delta_{i}^{h}+F_{i k} F_{j}^{h}-F_{j k} F_{i}^{h}+2 F_{i j} F_{k}^{h}\right)$

In view of equations (1.8) and (1.9), we have

We shall use the following:

$$
V_{i j k}^{h}=A_{i j k}^{h}-\frac{R}{(n+2)(n+4)}\left(g_{i k} \delta_{j}^{h}-g_{j k} \delta_{i}^{h}+F_{i k} F_{j}^{h}-F_{j k} F_{i}^{h}+2 F_{i j} F_{k}^{h}\right)
$$

DEFINITION (1.1): A Kaehler space is said to be recurrent space of second order , if it satisfies

$$
R_{i j k, a b}^{h}-\lambda_{a b} R_{i j k}^{h}=0,
$$

for some non-zero tensor $\lambda_{a b}$, and is called Ricci - recurrent of second order if it satisfies

Multiplying (1.12) by $g^{i j}$, we have

$$
R_{i j, a b}-\lambda_{a b} R_{i j}=0 \text {, }
$$

$$
R_{, a b}-\lambda_{a b} R=0 .
$$

REMARK (1.1) : From (1.11) and (1.12), it follows that every Kaehlerian recurrent space of second order is Kaehlerian - Ricci recurrent space of second order, but the converse is not true.

1). All latin indices run over the same range from 1 to $n$.

2). Numbers in square brackets refer to the references given at the end of the paper.

\section{Einstein - Kaehlerian Recurrent Spaces Of Second Order}

DEFINITION (2.1) : A Kaehler space satisfying the relation

$$
A_{i j k, a b}^{h}-\lambda_{a b} A_{i j k}^{h}=0,
$$

for some non -zero tensor $\lambda_{a b}$, will be called an Einstein - Kaehlerian Conharmonic recurrent space of second order or, briefly $A-K^{*}$ space.

DEFINITION (2.2): A Kaehler space satisfying the relation

$$
V_{i j k, a b}^{h}-\lambda_{a b} V_{i j k}^{h}=0,
$$

for some non- zero tensor $\lambda_{a b}$, will be called an Einstein - Kaehlerian space with recurrent Bochner curvature tensor of second order or, briefly a $V-K^{*}$ space.

THEOREM (2.1): A necessary and sufficient condition for an $A-K^{*}$ space to be a Kaehlerian recurrent space of second order is that the scalar curvature be equal to zero.

PROOF: Suppose that $A-K^{*}$ space is Kaehlerian recurrent space of second order. Making use of equations (1.7), (1.7a) and (1.9) in (2.1), we have

$$
R_{i j k, a b}^{h}=\lambda_{a b}\left[R_{i j k}^{h}+\frac{2 R}{n(n+4)}\left(g_{i k} \delta_{j}^{h}-g_{j k} \delta_{i}^{h}+F_{i k} F_{j}^{h}-F_{j k} F_{i}^{h}+2 F_{i j} F_{k}^{h}\right)\right]
$$

Since, an $A-K^{*}$ space is Kaehlerian recurrent space of second order, then equation (2.3) reduces to

$$
\frac{2 R}{n(n+4)}\left(g_{i k} \delta_{j}^{h}-g_{j k} \delta_{i}^{h}+F_{i k} F_{j}^{h}-F_{j k} F_{i}^{h}+2 F_{i j} F_{k}^{h}\right)
$$

which gives $R=0$.

Conversely, if a $A-K^{*}$ space satisfies $R=0$, then equation (2.3) reduces to

$$
R_{i j k, a b}^{h}-\lambda_{a b} R_{i j k}^{h}=0 \text {, }
$$

which shows that the space is Kaehlerian recurrent space of second order, which completes the proof.

Similarly, in view of theorem (2.2) and equations (1.7), (1.7a) and (1.8), we can prove the following theorem.

THEOREM (2.2): A necessary and sufficient condition for an Einstein - Kaehlerian space with recurrent Bochner curvature tensor of second order to be a Kaehlerian recurrent space of second order is that the scalar curvature be equal to zero.

THEOREM (2.3): An $A-K^{*}$ space is the Einstein- Kaehlerian space with recurrent Bochner curvature tensor iff the scalar curvature $R=0$.

PROOF: Suppose that an $A-K^{*}$ space is the Einstein- Kaehlerian space with recurrent Bochner curvature tensor of second order.

Differentiating (1.10) covariantly w.r.t. $x^{a}$, again differentiate the result thus obtained w.r.t. $x^{b}$, and then using the fact $R_{, a b}=0$, we get

$$
V_{i j k, a b}^{h}=A_{i j k, a b}^{h}
$$

Multiplying (1.10) by $\lambda_{a b}$ and subtracting from (2.6), we get 


$$
\begin{gathered}
V_{i j k, a b}^{h}-\lambda_{a b} V_{i j k}^{h}=A_{i j k, a b}^{h}-\lambda_{a b} A_{i j k}^{h}+\frac{\lambda_{a b} R}{(n+2)(n+4)}\left(g_{i k} \delta_{j}^{h}-\right. \\
\left.g_{j k} \delta_{i}^{h}+F_{i k} F_{j}^{h}-F_{j k} F_{i}^{h}+2 F_{i j} F_{k}^{h}\right)
\end{gathered}
$$

Now, making use of the above supposition, equation (2.7) reduces to

$$
\frac{\lambda_{a b} R}{(n+2)(n+4)}\left(g_{i k} \delta_{j}^{h}-g_{j k} \delta_{i}^{h}+F_{i k} F_{j}^{h}-F_{j k} F_{i}^{h}+2 F_{i j} F_{k}^{h}\right)=0
$$

which implies $R=0$.

Conversely, let us suppose that in an $A-K^{*}$ space, the scalar curvature $R=0$. Hence, equation (2.7) reduces to

$$
V_{i j k, a b}^{h}-\lambda_{a b} V_{i j k}^{h}=0 .
$$

which shows that the space is an Einstein- Kaehlerian space with recurrent Bochner curvature tensor of second order.

THEOREM (2.4): If a Kaehler space satisfies any two of the following properties:

(i) The space is an $A-K^{*}$ space,

(ii) The space is an $V-K^{*}$ space,

(iii) The scalar curvature is equal to zero; it must also satisfy the third.

PROOF: The Einstein- Kaehlerian conharmonic recurrent space of second order (i.e. $A-K^{*}$ ) space and Einstein- Kaehlerian space with recurrent Bochner curvature tensor of second order (i.e. $V-K^{*}$ ) are characterized respectively by equations (2.1) and (2.2).

The statement of the above theorem follows in view of equations (2.1), (2.2) and (2.7).

\section{Einstein- Kaehlerian Symmetric Spaces Of Second Order}

DEFINITION (3.1): A Kaehler space satisfying the condition

$$
R_{i j k, a b}^{h}=0 \text {, or, equivalently } R_{i j k l}, a b=0,
$$

is called Kaehlerian symmetric space of second order and it is called Kaehlerian Ricci- symmetric space of second order, if it satisfies

$$
R_{\ddot{y}, a b}=0,
$$

Multiplying (3.2) by $g^{\ddot{j}}$, we get

$$
R_{, a b}=0,
$$

REMARK (3.1): From (3.1) and (3.2), it follows that every Kaehlerian symmetric space of second order is Kaehlerian- Ricci symmetric space of second order, but the converse is not necessarily true.

DEFINITION (3.2): A Kaehler space satisfying the relation

$$
A_{\ddot{y} k, a b}^{h}=0 \text {, or , equivalently } A_{i j k l}, a b=0,
$$

is called an Einstein- Kaehlerian conharmonic symmetric space of second order.

DEFINITION (3.3): A Kaehler space satisfying the relation

$$
V_{\not j k, a b}^{h}=0 \text {, or, equivalently } V_{i j k}, a b=0,
$$

is called an Einstein- Kaehlerian space with symmetric Bochner curvature tensor of second order.

THEOREM (3.1): A necessary and sufficient condition for a $V-K^{*}$ space with symmetric Bochner curvature tensor of second order is that the space be Kaehlerian- symmetric space of second order.

PROOF: Differentiating (1.8) covariantly w.r.t. $x^{a}$, again differentiate the result so obtained w.r.t. $x^{b}$ and then using the fact $R_{, a b}=0$, we get

$$
V_{\ddot{j} k, a b}^{h}=R_{\ddot{j} k, a b}^{h}
$$

If the space is an Einstein- Kaehlerian space with symmetric Bochner curvature tensor of second order, then (3.6) reduces to

$$
R_{i j k, a b}^{h}=0,
$$

which shows that the space is Kaehlerian symmetric space of second order. Hence the condition is necessary.

Conversely, let the space be Kaehlerian symmetric space of second order, then (3.6) gives

$$
V_{i j k, a b}^{h}=0 \text {, }
$$

which shows that the space is Einstein- Kaehlerian space with symmetric Bochner curvature tensor of second order. Hence, the condition is sufficient.

This completes the proof of the theorem. 
THEOREM (3.2): A necessary and sufficient condition for an Einstein- Kaehlerian space to be EinsteinKaehlerian conharmonic symmetric space of second order is that the space be an Einstein- Kaehlerian space with symmetric Bochner curvature tensor of second order.

PROOF: Differentiating (1.10) covariantly w.r.t. $x^{a}$, again differentiate the result so obtained w.r.t. $x^{b}$, and then using the fact

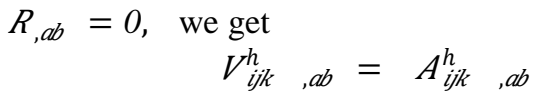

If the space is an Einstein- Kaehlerian conharmonic symmetric space of second order, then (3.7) in view (3.4) reduces to

$$
V_{i j k, a b}^{h}=0,
$$

which shows that the space is Einstein- Kaehlerian space with symmetric Bochner curvature tensor of second order. Hence the condition is necessary.

Conversely, let the space be an Einstein- Kaehlerian space with symmetric Bochner curvature tensor of second order, then (3.7), in view of (3.5) reduces to

$$
A_{i j k, a b}^{h}=0,
$$

which shows that the space is an Einstein- Kaehlerian conharmonic symmetric space of second order. Hence, the condition is sufficient.

This completes the proof of the theorem.

THEOREM (3.3): For every Einstein- Kaehlerian conharmonic symmetric space of second order, which is an Einstein- Kaehlerian space with symmetric Bochner curvature tensor of second order, the relation

$$
V_{i j k}^{h}-A_{i j k}^{h}+\frac{R}{(n+2)(n+4)}\left(g_{i k} \delta_{j}^{h}-g_{j k} \delta_{i}^{h}+F_{i k} F_{j}^{h}-F_{j k} F_{i}^{h}+2 F_{i j} F_{k}^{h}\right)=0
$$

PROOF: If the space is an Einstein- Kaehlerian conharmonic symmetric space of second order as well as Einstein- Kaehlerian space with symmetric Bochner curvature space of second order, then (2.7) in view of (3.4) and (3.5) reduces to the required relation (3.8).

\section{References}

[1] Matsumoto, M. : On Kaehlerian spaces with parallel or vanishing Bochner curvature tensor, Tensor, N. S. , 20(1), 25- 28 (1969).

[2] Negi, D. S. and Rawat, K. S. : Some bi- recurrence and bi- symmetric properties in a Kaehlerian space, Acta Cien. Ind., Vol. XX,M, No.1, 95-100 (1994).

[3] Rawat, K. S. and Gyan Prakash : Some recurrence and symmetric properties of a Kaehlerian space, Acta Ciencia Indica, Vol. XXX M, No.4, 701-704 (2004).

[4] Rawat, K. S. and Girish Dobhal : On the bi- recurrent Bochner curvature tensor, Jour. of the tensor society, Vol.1, 33-40 (2007).

[5] Rawat, K. S. and Kunwar Singh : Some bi- recurrence properties in a Kaehlerian space, Jour. PAS , Vol. 14 (Mathematical Science) pp. 199-205 (2008).

[6] Rawat, K. S. and Girish Dobhal : Study of the decomposition of recurrent curvature tensor fields in a Kaehlerian recurrent space, Jour. Pure and Applied Mathematica Sciences, Vol. LXVIII, No. 1-2, Sept (2008).

[7] Rawat, K. S. and Virendra Prasad : Some recurrent and symmetric properties in an almost Kaehlerian space, Jour. PAS, Vol. 14 (Mathematical Sciences), pp. 283-288 (2008).

[8] Rawat, K. S. and G. P. Silswal: Theory of Lie-derivatives and motions in Tachibana spaces, News Bull. Cal. Math. Soc. , 32, (13), 15-20 (2009).

[9] Rawat K. S. and Mukesh Kumar: On curvature collineations in a Tachibana recurrent space, Aligarh Bull. Math., 28 No. 1-2, 6369 (2009) MR 2769016.

[10] Rawat, K. S. and Virendra Prasad: On holomorphically projectively flat parabolically Kaehlerian spaces, Rev. Bull. Cal. Math. Soc. , 18, (1), 21-26(2010).

[11] Rawat, K. S. and Nitin Uniyal: On infinitesimal conformal and projective transformations of K-space and Kaehlerian recurrent space, International Trans. in Mathematical Science and Comp. , Vol. 3, No. 2, 205-213 (2010).

[12] Rawat, K. S. and Girish Dobhal: The study of Tachibana bi-recurrent spaces, Antarctica Jour. Math. , 7(4), 413-420(2010).

[13] Rawat, K. S. and Mukesh Kumar: On hyper surfaces of a conformally Flat Kaehlerian recurrent space, Pure and Applied Mathematica Sciences, Vol. LXXIII, No. 1-2, 7-13 (2011).

[14] Rawat, K. S. and Nitin Uniyal: On conformal Transformation in an almost Kaehlerian and Kaehlerian spaces, Jour. of Progressive Science, Vol. 2, No. 2, 138-141 (2011).

[15] Roter, W. : A note on second order recurrent spaces, Bull. Acad. Polon. Sci. Scr. Math. Astronom. Phys. 12, 621-626 (1964).

[16] Singh, S. S. : On a Kaehlerian space with recurrent holomorphic projective curvature tensor, Acc. Naz. Dei Lincei, Rend, 55 (3-4), 214-218 (1973).

[17] Singh, S. S. : On Kaehlerian recurrent and Ricci- recurrent spaces of second order, Atti della Accademaia delle Scienze di Torino, $106,11,509-518(1972)$

[18] Sinha, B.B. : On H- curvature tensors in a Kaehlerian manifold , Kuyangpook Math. Journ. 13(2), 185-189(1973).

[19] Tachibana, S. : On Bochner curvature tensor, Nat. Sci. Rep., Ochanomizu Univ. 18(1), 15-19(1967).

[20] Yano, K. : Differential Geometry On Complex and almost Complex spaces, Pergamon Press (1965). 